\title{
Pediatric non alcoholic fatty liver disease: more on novel treatment targets
}

\author{
Pietro Vajro*, Giulia Paolella, Marco Poeta, Cristina Pizza, Maria Sangermano and Grazia Massa
}

\begin{abstract}
The mainstay treatment of non alcoholic fatty liver disease (NAFLD) based on weight loss and/or lifestyle changes is most often unsuccessful at all ages, thus requiring the implementation of pharmacological strategies. Targeting insulin resistance and oxidative stress has recently proven unsatisfactory. Among a number of proposed innovative approaches targeting novel pathomechanisms, probiotics appear an interesting and reasonable option acting on gut-liver axis malfunction through the modulation of diet-driven, obesogenic, and inflammatory intestinal microbiota.

A combined multiple pharmacological therapy directed simultaneously towards novel and old pathomechanisms (including, e.g., insulin resistance, oxidative stress, gut-liver axis, apoptosis) along with lifestyle interventions however might be necessary both in adult and pediatric NAFLD therapy.
\end{abstract}

Keywords: Non alcoholic fatty liver disease, Children, Gut-liver axis, Microbiota, Probiotics, Therapy

\section{Commentary}

The available treatment options for NAFLD at all ages are currently still far from being satisfactory. Therefore we read with much interest the article by Giorgio et al. which appeared most recently in the Journal [1]. The Authors gave pediatricians a thorough update on several aspects of NAFLD pathomechanisms, which were reviewed also under the light of potential treatments targets. Dr Giorgio et al. should be congratulated in particular for the attention paid also to the intestinal microbiota and the gut-liver axis. These issues in fact only recently emerged as additional, possibly pivotal, protagonists in the busy scenario of NAFLD pathogenesis not only in animal models [2] but also in humans, including childhood [3]. This now begins to appear paramount due to intestinal microbiota's susceptibility of being modulated by a number of agents (including pre-, pro-, and syn-biotics) [4].

Since the 2007 Cochrane meta-analysis [5], which could neither suggest nor refute the use of probiotics as therapeutic option for patients with NAFLD and nonalcoholic steatohepatitis (NASH), most recently at least two double blind, controlled, pilot RCTs (both with

\footnotetext{
* Correspondence: pvajro@unisa.it
Department of Medicine and Surgery, University of Salerno, Via S. Allende,

* Correspondence: pvajro@unisa.it
Department of Medicine and Surgery, University of Salerno, Via S. Allende, 84081, Baronissi -Salerno, Italy
}

(c) 2013 Vajro et al.; licensee BioMed Central Ltd. This is an Open Access article distributed under the terms of the Creative Commons Attribution License (http://creativecommons.org/licenses/by/2.0), which permits unrestricted use, distribution, and reproduction in any medium, provided the original work is properly cited. probiotics as the sole treatment) became available in the inary good results upon aminotransferases activity in adults [6] and, even more, in pediatric population where also a number of markers of intestinal dysbiosis and/or gut-liver axis malfunction appeared to be modulated by probiotic treatment [7]. Hence, these results, altogether, appear to nicely fit the intense network existing between gut and liver and probiotics influence $[3,4]$ which also Giorgio et al. mentioned in their study [1]. Moreover they may add further strength to the Authors' view that large, well conducted, controlled studies with probiotics or other therapeutic tools able to interact with the gut microbiota, alone and/or together with other therapeutic measures (i.e. "multi-target therapy" avoiding the monotherapeutic direction approach) $[4,8]$, appear fully justified also in pediatric NAFLD.

At the same time, for future NAFLD therapeutic studies, one should underline the importance of warning on the strict monitoring patients' anthropometric changes to avoid under/over-estimation of the effects of the therapies under consideration. Unpredictable concurrent individual lifestyle changes in fact represent a relevant confounding factor which has hitherto flawed a number of medium and long-term studies in both pediatric and adult obese populations [4]. Accordingly, also the categorical need 
for the assessment of "biopsy proven" basal and/or follow-up histological liver damage should be cautiously reconsidered. In addition to the unpredictability of sampling error potentials, one should bear in mind that liver biopsy results may in fact represent only a transient \& mutable snapshot during the extremely irregular lifestyle- and diet-driven hepatic histological changes, especially in the pediatric age.

\section{Abbreviations}

(NAFLD): Non alcoholic fatty liver disease; (NASH): Non-alcoholic steatohepatitis.

\section{Competing interests}

The authors declare that there is no competing interest regarding the material discussed in the manuscript.

\section{Authors' contributions}

PV and GP -prepared the first draft of manuscript. MP, CP, MS, and GM- prepared bibliographical background. PV - was the guarantor of manuscript, supervised, revised the manuscript. All authors read and approved the final manuscript.

\section{Acknowledgments}

The Authors thank the University of Salerno for the Grant FARB-ex 60\% 2012 to PV.

Received: 21 May 2013 Accepted: 18 July 2013

Published: 19 July 2013

\section{References}

1. Giorgio V, Prono F, Graziano F, Nobili V: Pediatric non alcoholic fatty liver disease: old and new concepts on development, progression, metabolic insight and potential treatment targets. BMC Pediatr 2013. in press.

2. Esposito E, lacono A, Bianco G, Autore G, Cuzzocrea S, Vajro P, Canani RB, Calignano A, Raso GM, Meli R: Probiotics reduce the inflammatory response induced by a high-fat diet in the liver of young rats. J Nutr 2009, 139:905-911.

3. Vajro P, Paolella G, Fasano A: Microbiota and gut-liver axis: a mini-review on their influences on obesity and obesity related liver disease. J Pediatr Gastroenterol Nutr 2013, 56:461-468.

4. Vajro P, Lenta S, Pignata C, Salerno M, D'Aniello R, De Micco I, Paolella G, Parenti G: Therapeutic options in pediatric non alcoholic fatty liver disease: current status and future directions. Ital J Pediatr 2012, 38:55.

5. Lirussi F, Mastropasqua E, Orando S, Orlando R: Probiotics for non-alcoholic fatty liver disease and/or steatohepatitis. Cochrane Database Syst Rev 2007:CD005165.

6. Aller R, De Luis DA, Izaola O, Conde R, Gonzalez Sagrado M, Primo D, De La Fuente B, Gonzalez J: Effect of a probiotic on liver aminotransferases in nonalcoholic fatty liver disease patients: a double blind randomized clinical trial. Eur Rev Med Pharmacol Sci 2011, 15:1090-1095.

7. Vajro P, Mandato C, Licenziati MR, Franzese A, Vitale DF, Lenta S, Caropreso M, Vallone G, Meli R: Effects of Lactobacillus rhamnosus strain GG in pediatric obesity-related liver disease. J Pediatr Gastroenterol Nutr 2011, 52:740-743

8. Polyzos SA, Kountouras J, Zavos C, Deretzi G: Nonalcoholic fatty liver disease: multimodal treatment options for a pathogenetically multiplehit disease. J Clin Gastroenterol 2012, 46:272-284.

doi:10.1186/1471-2431-13-109

Cite this article as: Vajro et al:: Pediatric non alcoholic fatty liver disease: more on novel treatment targets. BMC Pediatrics 2013 13:109.

\section{Submit your next manuscript to BioMed Central and take full advantage of:}

- Convenient online submission

- Thorough peer review

- No space constraints or color figure charges

- Immediate publication on acceptance

- Inclusion in PubMed, CAS, Scopus and Google Scholar

- Research which is freely available for redistribution 